\title{
Article
}

\section{Self-monitoring for improving control of blood pressue in patients with hypertension}

Qamar, N, Bray, Emma, Glynn, L G, Fahey, T, Mant, J, Holder, R L and McManus, $\mathrm{R}$

Available at http://clok.uclan.ac.uk/9558/

Qamar, N, Bray, Emma ORCID: 0000-0001-9882-3539, Glynn, L G, Fahey, T, Mant, J, Holder, $R$ L and McManus, $R$ (2013) Self-monitoring for improving control of blood pressue in patients with hypertension. The Cochrane Collaboration (1).

It is advisable to refer to the publisher's version if you intend to cite from the work. http://dx.doi.org/10.1002/14651858.CD010311

For more information about UCLan's research in this area go to http://www.uclan.ac.uk/researchgroups/ and search for <name of research Group>.

For information about Research generally at UCLan please go to http://www.uclan.ac.uk/research/

All outputs in CLoK are protected by Intellectual Property Rights law, including Copyright law. Copyright, IPR and Moral Rights for the works on this site are retained by the individual authors and/or other copyright owners. Terms and conditions for use of this material are defined in the policies page.

\section{CLoK}

Central Lancashire online Knowledge www.clok.uclan.ac.uk

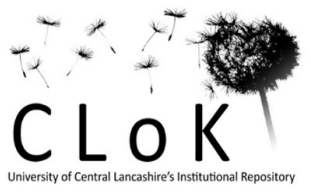




\title{
Self-monitoring for improving control of blood pressure in patients with hypertension (Protocol)
}

\author{
Qamar N, Bray EP, Glynn LG, Fahey T, Mant J, Holder RL, McManus R
}

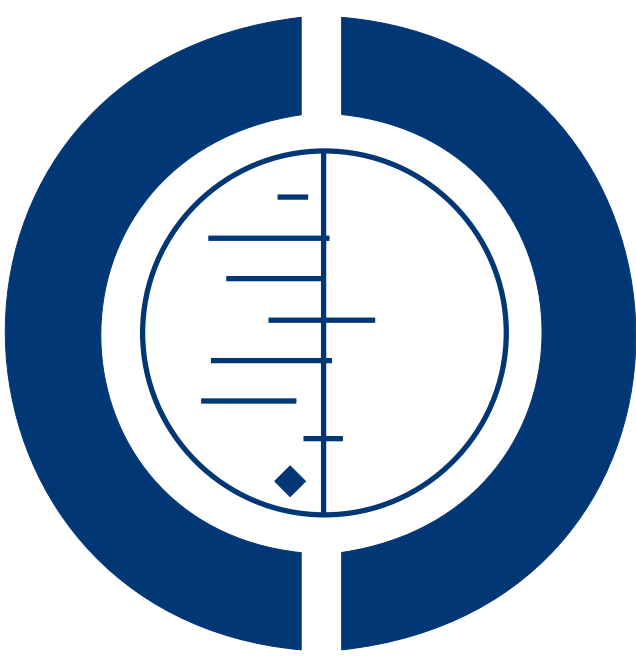

\section{THE COCHRANE COLLABORATION $^{\circledR}$}

This is a reprint of a Cochrane protocol, prepared and maintained by The Cochrane Collaboration and published in The Cochrane Library 2013, Issue 1

http://www.thecochranelibrary.com

\section{WILEY}


TABLE OF CONTENTS

HEADER . . . . . . . . . . . . . . . . . . . . . . . . . . . . . . . . . . . . 1

ABSTRACT . . . . . . . . . . . . . . . . . . . . . . . . . . . . . . . . . . . . . . . . . . . . .

BACKGROUND . . . . . . . . . . . . . . . . . . . . . . . . . . . . . . . . . . . .

OBJECTIVES . . . . . . . . . . . . . . . . . . . . . . . . . . . . . . . . . . . . . . . . . . . .

METHODS . . . . . . . . . . . . . . . . . . . . . . . . . . . . . . . . . . . . . .

ACKNOWLEDGEMENTS . . . . . . . . . . . . . . . . . . . . . . . . . . . . . . . . . . . . . . . .

REFERENCES . . . . . . . . . . . . . . . . . . . . . . . . . . . . . . . . . . . . . . 4

APPENDICES . . . . . . . . . . . . . . . . . . . . . . . . . . . . . . . . . . . . . . 5

CONTRIBUTIONS OF AUTHORS . . . . . . . . . . . . . . . . . . . . . . . . . . . . . . . . . . . . . . . . .

DECLARATIONS OF INTEREST . . . . . . . . . . . . . . . . . . . . . . . . . . . . . . . . . . . 6

SOURCES OF SUPPORT . . . . . . . . . . . . . . . . . . . . . . . . . . . . . . . . . . . . . . . . 


\title{
[Intervention Protocol]
}

\section{Self-monitoring for improving control of blood pressure in patients with hypertension}

\author{
Nashat Qamar ${ }^{1}$, Emma P Bray $^{1}$, Liam G Glynn ${ }^{2}$, Tom Fahey ${ }^{3}$, Jonathan Mant ${ }^{4}$, Roger L Holder ${ }^{1}$, Richard McManus ${ }^{5}$ \\ ${ }^{1}$ Primary Care Clinical Sciences, University of Birmingham, Birmingham, UK. ${ }^{2}$ Department of General Practice, National University \\ of Ireland, Galway, Ireland. ${ }^{3}$ Department of Family Medicine and General Practice, Royal College of Surgeons in Ireland Medical \\ School, Dublin, Ireland. ${ }^{4}$ General Practice \& Primary Care Research Unit, Department of Public Health \& Primary Care, University \\ of Cambridge, Cambridge, UK. ${ }^{5}$ Department of Primary Care Health Sciences, University of Oxford, Oxford, UK \\ Contact address: Nashat Qamar, Primary Care Clinical Sciences, University of Birmingham, Vincent drive, Birmingham, B15 2TTT, \\ UK. nashat.qamar@nhs.net.nashatqamar@doctors.net.uk.
}

Editorial group: Cochrane Hypertension Group.

Publication status and date: New, published in Issue 1, 2013.

Citation: Qamar N, Bray EP, Glynn LG, Fahey T, Mant J, Holder RL, McManus R. Self-monitoring for improving control of blood pressure in patients with hypertension. Cochrane Database of Systematic Reviews 2013, Issue 1. Art. No.: CD010311. DOI: 10.1002/14651858.CD010311.

Copyright (C) 2013 The Cochrane Collaboration. Published by John Wiley \& Sons, Ltd.

\begin{abstract}
A B S T R A C T
This is the protocol for a review and there is no abstract. The objectives are as follows:

The objective of this review is to determine the effect of SBPM in adults with hypertension on blood pressure control as compared to OBPM or usual care.
\end{abstract}

\section{B A C K G R O U N D}

Hypertension has been well recognised for many years as one of the most important modifiable risk factors in the prevention of stroke and cardiovascular disease (MacMahon 1990). Globally the public health burden of the disease continues to be a significant problem with an increasingly aging population (Kearney 2005). However, despite this, blood pressure control among hypertensive patients remains poor with only a minority of people treated to satisfactory levels (Wolf-Maier 2004).

Due to the low cost and broad availability of validated electronic BP devices (O’Brien 2010), self blood pressure monitoring (SBPM) at home by patients is increasingly common and is regarded as a more reliable and reproducible representation of blood pressure control due to minimisation of the white coat effect (Stergiou 2002). It has been recommended for the evaluation of hypertension (NICE 2011) as well as a tool for increasing the likelihood of therapeutic intensification in response to above target blood pressure readings (Agarwal 2011).

SBPM empowers patients; it is cost effective (McManus 2005), well tolerated and has been shown to be a better predictor of end organ damage than traditional office blood pressure monitoring (OBPM) (Bobrie 2004).

\section{Why it is important to do this review}

The most recent Cochrane review on interventions to improve hypertension management (Glynn 2010) proved too large for timely updates. It classified 72 trials of interventions, based on searches from 2008, into six categories: self monitoring, patient education, health professional education, health professional led care (nurse 
and/or pharmacist), appointment reminder systems and organisational interventions. The review concluded that self-monitoring and appointment reminders may be useful adjuncts to antihypertensive drug therapy implemented by means of a vigorous stepped care approach, but required further evaluation.

Since then there has been an increasing body of literature which pertains to the beneficial effect of home blood pressure monitoring on blood pressure control in hypertensive patients (Bray 2010 - 25 trials) (Agarwal 2011 - 37 trials) (Uhlig 2012 - 49 trials). The aim of this review is to provide an up-to-date assessment on the effectiveness of self-monitoring in the management of hypertension. A previous version of this review (Bray 2010) requires updating due to new studies in the last two years relating to the effects of home blood pressure monitoring.

\section{O B J E C T I VES}

The objective of this review is to determine the effect of SBPM in adults with hypertension on blood pressure control as compared to OBPM or usual care.

\section{METHODS}

\section{Criteria for considering studies for this review}

\section{Types of studies}

Randomised controlled trials (RCTs) are eligible if the intervention group includes home or self blood pressure measurement, if the usual care or control group does not include home/self-monitoring and if a blood pressure outcome measure is available which has been taken independently of the self measurement. These are usually either systolic or diastolic office BP readings or mean daytime ambulatory BP readings. Trials will be considered that include self-monitoring as a sole intervention or as an adjunct to other interventions (e.g. education, nurse/ pharmacist support, telemonitoring, etc).

\section{Types of participants}

The participants of interest are adult patients (18 years or over) with a diagnosis of primary hypertension (either treated or not treated with antihypertensive medications) assessed in a primary care, outpatient or community setting.

\section{Types of interventions}

The intervention of interest is home or self monitoring of blood pressure in patients with hypertension.

\section{Types of outcome measures}

\section{Primary outcomes}

Studies will be included if they report:

1. Change in mean office systolic blood pressure (SBP) and/or mean office diastolic blood pressure (DBP)

2. Change in mean systolic and diastolic ambulatory blood pressure $(\mathrm{ABP})$

3. The proportion of participants achieving target $\mathrm{BP}$ in each group (control as defined by each randomised trial's investigators)

\section{Secondary outcomes}

Additional secondary outcomes that will be looked at are:

- 'Therapeutic intensification' - defined as the addition of another antihypertensive agent, increasing dosage of medication or both in response to above target blood pressure readings (this outcome will be assessed by subgroup analysis of treated and untreated patients)

- Adverse events in the self monitoring and control groups including any evidence of mortality or cardiovascular morbidity

- Adherence to intervention (drop out rate)

- Adherence to medication (compliance rate)

- Health related quality of life data related to self-monitoring of blood pressure

- Other surrogate markers such as LVH (left ventricular hypertrophy) or LV mass

\section{Search methods for identification of studies}

\section{Electronic searches}

The following electronic databases will be searched: The Hypertension Group specialised register (1946 to October 2012), the Cochrane Central Register of Controlled Trials (CENTRAL) 2012 Issue 10, the Hypertension Group specialised register (1946 to October 2012), MEDLINE (2009 to October 2012), and EMBASE (2009 to October 2012). The MEDLINE search strategy (Appendix 1) will be translated into the other databases using the appropriate subject headings and syntax. 


\section{Searching other resources}

Information from grey literature sources, such as internal reports and conference proceedings will be sought. The references of all retrieved archives will be screened and experts in the field contacted for potentially relevant trials or unpublished material of systematic reviews and meta-analyses. In addition we will maintain dialogue and share results with the authors of collaborating teams from the split of the existing review.

\section{Data collection and analysis}

Two separate authors (NQ and EB) will independently assess the list of citations and abstracts generated from the searches for eligibility.

\section{Selection of studies}

Studies that are clearly irrelevant will be excluded on the basis of their titles if possible. The remaining abstracts will be reviewed and identified as potentially relevant, not relevant or uncertain. Potentially relevant and uncertain articles will be retrieved in full when a final decision will be made on eligibility by two independent reviewers. Disagreements on classification will be discussed and arbitrated by a third author (RJM). No additional methodological quality criteria including study size or language of publication will be applied.

\section{Data extraction and management}

Data extraction will occur independently by two authors (NQ and EB) regarding study design, methods, clinicians and patients, interventions, outcomes and potential sources of bias using a structured data collection pro forma. Where studies report outcomes at more than one time point (e.g. 6 and 12 months), data concerning the longest follow up will be extracted. Again any differences in the interpretation of the data will be resolved by discussion and arbitration with a third author (RJM). Where data are found to be missing, or further enquiries needed, the original authors of the studies will be contacted.

\section{Assessment of risk of bias in included studies}

For assessment of study quality and reporting bias, we will use the Cochrane Collaboration 'Risk of Bias' (ROB) assessment tool to record and describe the methods used in randomisation sequence generation, allocation concealment, blinding of participants for each main outcome and class of outcomes. We will also use the $\mathrm{ROB}$ tool to assess attrition bias by looking at the completeness of outcome data for each main outcome including the number of exclusions in each intervention group (compared to the total participants randomised) and whether these were reported and included in the analysis. Reporting bias due to selective outcome reporting by the review authors will be examined as well as other sources of bias. This process of assessing bias will be carried out independently by two separate authors (NQ and EB).

\section{Measures of treatment effect}

Weighted mean differences (WMD) will be calculated for the overall change in systolic and diastolic blood pressure (both for office and $A B P M)$ between intervention and control. Depending upon the measurement indices used in individual studies, Relative Risk (RR) or Odds Ratio (OR) will be the preferred choice when reporting other primary and secondary outcomes. Risk Difference (RD) may also be a relevant measure of treatment effect for some of the outcomes.

\section{Unit of analysis issues}

Care will be taken to avoid problematic unit of analysis issues if the same group of participants are included twice in the same meta-analysis. Where studies including more than one intervention group with a single comparator arm are used, both intervention groups will be included and the number of patients split across the interventions arms.

\section{Dealing with missing data}

Any potential missing data will be discussed by the authors of this review and if necessary clarified by corresponding with the relevant study authors. All participants not receiving the assigned intervention according to the protocol as well as those dropping out or lost to follow up, will be included in the analysis on an intention to treat basis. This will be performed, with sensitivity analysis, by either available case analysis or imputation (see section 16.2.3 of Cochrane Handbook). In looking at the primary outcomes, where the standard deviation (SD) of the change in BP is not reported, this will be estimated using elementary theory of differences of correlated variables from the SD at baseline and final measurement (as per Bray 2010, p2, appendix 1).

\section{Assessment of heterogeneity}

Clinical heterogeneity will be assessed qualitatively by comparing the study characteristics of included trials and quantified using the chi-square test of systematic variation and the $\mathrm{I}^{2}$ statistic. Heterogeneity will be explored with sensitivity analysis by excluding single outlying results or restricting analysis to studies at low risk of bias. This will be further analysed using meta-regression with backward elimination to assess the associations between the treatment effect and the study characteristics (see below). Pooled data will only be reported where heterogeneity is not statistically significant $(\mathrm{P}>0.05)$. In addition heteroscedasticity between studies 
will be assessed and possible contributing factors will be investigated. This will be done using specialist statistical software such as STATA or SPSS.

\section{Assessment of reporting biases}

Reporting bias will be considered where studies do not report absolute BP measures at follow up in favour of indirect measures (e.g. change from baseline, achievement of target). Publication bias will be assessed by producing funnel plots of effect size and of sample size against WMD to provide a visual review of any potential bias

\section{Data synthesis}

Pooling and analysis of data will be carried out with RevMan 5. Separate analyses will be conducted for each intervention and outcome measure compared to usual care. Intervention effects will be calculated as relative risks with $95 \%$ confidence intervals for dichotomous data. For continuous data, we will calculate mean differences and WMDs with 95\% confidence intervals using a conservative fixed-effects meta-analysis model in the absence of significant heterogeneity ( $\mathrm{p}>0.05$ or $\mathrm{I}^{2}<50 \%$ ), or a random-effects model if heterogeneity is present.

In addition, the quality of the body of the evidence will be analysed according to the GRADE system as set out in chapter 12.2.1 of the Cochrane Handbook. This will take into account the methodological quality, directness of evidence, heterogeneity, precision of effect estimates and risk of publication bias. See below for example of Summary of Findings table.

\section{Subgroup analysis and investigation of heterogeneity}

The primary analysis will include all trials. Meta-regression will be used to investigate the effects of different study characteristics on treatment effects. Planned subgroups will include terms for age (continuous variable), sex of participants (male or female), length of follow up (continuous variable), number of treated or untreated patients (all treated, all untreated or mix of treated and untreated), mean baseline diastolic blood pressure (categorised to nearest $5 \mathrm{mmHg}$ ) and use of additional co-interventions (where these are part of the intervention in addition to self-monitoring). The variables that will be analysed continuously will not be categorised in advance in order to increase the power of the metaregression and more reliably allow determination of the incremental effects of these variables. Different studies include different age groups of patients and length of follow-up; hence, using arbitrary cut-off points may introduce an added source of bias. In case of non linear relationships between these variables and outcomes not detected by meta-regression as well as to further explore relationships where detected, these prespecified variables will be further investigated in an exploratory analysis examining the individual categories (quintiles in the case of continuous variables), in order to elucidate the nature of this relationship.

\section{Sensitivity analysis}

Sensitivity analysis will be performed to assess the impact of each study on the overall outcome with recalculation of both WMDs and meta-regression with the removal of each study one at a time from the analysis.

\section{ACKNOWLEDGEMENTS}

The authors wish to thank all members of the Cochrane Hypertension Group involved in facilitating this review as well as Douglas Salzwedel, Trials Search Coordinator for the Cochrane Hypertension Group, for his help in preparing the search strategy.

\section{R E F E R N C E S}

\section{Additional references}

\section{Agarwal 2011}

Agarwal, Rajiv, Bills, Jennifer E, Hecht, Tyler J.W, Light, Robert P. Role of Home Blood Pressure Monitoring in Overcoming Therapeutic Inertia and Improving Hypertension Control: A Systematic Review and MetaAnalysis. Hyoertension 2011;57:29-38.

Bobrie 2004

Bobrie G, Chatellier G, Genes N, Clerson P, Vaur L, Vaisse B, Menard J, Mallion JM. Cardiovascular prognosis of "masked hypertension" detected by blood pressure selfmeasurement in elderly treated hypertensive patients. JAMA 2004;291:1342-1349.
Bray 2010

Bray EP, Holder R, Mant J, McManus RJ. Does selfmonitoring reduce blood pressure? Meta-analysis with meta-regression of randomized controlled trials. Annals of Medicine 2010;42(5):371-86.

Glynn 2010

Glynn LG, Murphy AW, Smith SM, Schroeder K, Fahey T. Interventions used to improve control of blood pressure in patients with hypertension. Cochrane Database of Systematic Reviews 2010, Issue 3. [DOI: 10.1002/ 14651858.CD005182.pub4]

Kearney 2005

Kearney PM, Whelton M, Reynolds K, Muntner P, 
Whelton PK, He J. Global burden of hypertension: analysis of worldwide data. Lancet 2005;365:217-23.

\section{MacMahon 1990}

MacMahon S, Peto R, Cutler J, Collins R, Sorlie P, Neaton J, Abbott R, Godwin J, Dyer A, Stamler J. Blood pressure, stroke, and coronary heart disease I prolonged differences in blood pressure. prospective observational studies corrected for the regression dilution bias. Lancet 1990;335:765-774.

McManus 2005

McManus RJ, Mant J, Roalfe A, Oakes RA, Bryan S, Pattison HM, Hobbs FD. Targets and self monitoring in hypertension: randomised controlled trial and cost effectiveness analysis. BMJ 2005;331:493-496.

\section{NICE 2011}

National Institute for Health and Clinical Excellence. Hypertension: Clinical management of primary hypertension in adults. August 2011. NICE.

\section{O'Brien 2010}

O'Brien E, Atkins N, Stergiou G, Karpettas N, Parati G, Asmar R, Imaif Y, Wang J, Mengden T, Shennan A, European Society of Hypertension International Protocolrevision 2010 for the validation of blood pressure measuring devices in adults. Blood Pressure Monitoring 2010;15:23-38.

\section{Stergiou 2002}

Stergiou GS, Baibas NM, Gantzarou AP, Skeva II, Kalkana CB, Roussias LG, Mountokalakis TD. Reproducibility of home, ambulatory, and clinic blood pressure: implications for the design of trials for the assessment of antihypertensive drug efficacy. American Journal of Hypertension 2002;15: 101-104.

\section{Uhlig 2012}

Uhlig K, Balk EM, Patel K, Ip S, Kitsios GD, Obadan NO, Haynes SM, Stefan M, Rao M, Kong Win Chang L, Gaylor J, Iovin RC. Self-Measured Blood PressureMonitoring: Comparative Effectiveness. Comparative Effectiveness Review No. 45. (Preparedby the Tufts Evidence-based Practice Center under Contract No. HHSA 290-2007-10055I.)AHRQ Publication No. 12-EHCOO2-EF January 2012; 12:290-2007.

\section{Wolf-Maier 2004}

Katharina Wolf-Maier, Richard S. Cooper, Holly Kramer, José R. Banegas, Simona Giampaoli, Michel R. Joffres, et al.Hypertension Treatment and Control in Five European Countries, Canada, and the United States. Hypertension 2004;43(1):10-17.

\section{References to other published versions of this review}

\author{
Bray et al 2010 \\ Bray EP, Holder R, Mant J, McManus RJ. Does self- \\ monitoring reduce blood pressure? Meta-analysis with \\ meta-regression of randomized controlled trials. Annals of \\ Medicine 2010;42(5):371-386. \\ * Indicates the major publication for the study
}

\section{APPENDICES}

\section{Appendix I. Medline Search Strategy}

Database: Ovid MEDLINE(R) 1946 to Present with Daily Update

1 blood pressure monitoring, ambulatory/

2 ((blood pressure or bp) adj3 (24h or $24 \mathrm{hr}$ ? or 24 -h or $24-\mathrm{hr}$ ? or 24 hour? or ambulatory or determin $\$$ or measur $\$$ or monitoring or monitor\$ or self-measur\$ or self-monitor\$)).tw.

3 or $1-2$

4 (home or self\$).tw.

5 (telemedicine or tele-medicine or telemonitor $\$$ or tele-monitor $\$$ ).mp.

6 or/ $4-5$

7 randomized controlled trial.pt.

8 controlled clinical trial.pt.

9 randomized.ab.

10 placebo.ab.

11 drug therapy.fs.

12 randomly.ab.

13 trial.ab.

Self-monitoring for improving control of blood pressure in patients with hypertension (Protocol)

Copyright $\odot 2013$ The Cochrane Collaboration. Published by John Wiley \& Sons, Ltd. 
14 groups.ab.

15 or/ $7-14$

16 animals/ not (humans/ and animals/)

1715 not 16

183 and 6 and 17

\section{CONTRIBUTIONSOFAUTHORS}

- N Qamar: drafted and takes responsibility for the protocol. For the review will undertake study selection, data extraction, analysis and interpretation, and draft the manuscript.

- E P Bray: contributed to the protocol. For the review will undertake study selection, data extraction and contribute to the manuscript.

- L G Glynn: contributed to the protocol. For the review will be involved in interpretation, and contribute to the manuscript.

- T Fahey: contributed to the protocol. For the review will be involved in interpretation, and contribute to the manuscript.

- J Mant: contributed to the protocol. For the review will be involved in interpretation, and contribute to the manuscript.

- R Holder: contributed to the protocol. For the review will be involved in data extraction, statistical analysis, and contribute to the manuscript.

- R J McManus: contributed to the protocol. For the review will be involved in study selection, data extraction, interpretation, and contribute to the manuscript. Will act as guarantor for the final output.

\section{DECLARATIONSOF INTEREST}

Nashat Qamar, Emma P Bray, Liam G Glynn, Tom Fahey, Roger L Holder: None Known.

Richard McManus is supported by an NIHR Career Development Fellowship and has received support for research in terms of blood pressure monitors from Omron and Lloyds Pharmacies.

Jonathan Mant has done consultancy work for Boehringer Ingelheim, Pharmaswiss and Expert-24.

\section{SOURCES OF SUPPORT}




\section{Internal sources}

- NIHR, UK

This work is funded by a National Institute of Health Research (NIHR) In Practice Fellowship grant. The views and opinions expressed in this paper are those of the authors and not necessarily those of the NHS, the NIHR or the Department of Health.

\section{External sources}

- No sources of support supplied 